\title{
SARS-CoV-2 infections among neonates born to women with SARS-CoV-2 infection: maternal, pregnancy and birth characteristics
}

Emily O’Malley Olsen ( $\sim$ eocevent397@cdc.gov )

Centers for Disease Control and Prevention

Nicole M. Roth

Centers for Disease Control and Prevention

Kathryn Aveni

New Jersey Department of Health

Pauline Santos

New York State Department of Health

Lindsey Sizemore

Tennessee Department of Health

Umme-Aiman Halai

Los Angeles County Department of Public Health

Eirini Nestoridi

Massachusetts Department of Public Health

Similoluwa Sowunmi

California Department of Public Health

Jerusha Elana Barton

Georgia Department of Public Health

Evan Mobley

Missouri Department of Public Health

Samantha Siebman

Minnesota Department of Health

Chris Fussman

Michigan Department of Health and Human Services

Deborah Mbotha

Washington State Department of Health

Paula Dzimira

Pennsylvania Department of Health

Kristin M. Silcox

Maryland Department of Health

Salma Khuwaja 
Houston Health Department

\section{Danacamile Roscom}

High Sierra Area Health Education Center

Mamie Lush

Nebraska Department of Health and Human Services

\section{Sarah Chicchelly}

Kansas Department of Health and Environment

\section{Camille Delgado-López}

Puerto Rico Department of Health

\section{Levi Schlosser}

North Dakota Department of Health

Jennifer S. Read

Vermont Department of Health

\section{Sascha R. Ellington}

Centers for Disease Control and Prevention

Aron J. Hall

Centers for Disease Control and Prevention

\section{Suzanne M. Gilboa}

Centers for Disease Control and Prevention

\section{Van T. Tong}

Centers for Disease Control and Prevention

\section{Kate R. Woodworth}

Centers for Disease Control and Prevention

\section{Short Report}

Keywords: pregnancy, COVID-19, SARS-CoV-2, perinatal infection

Posted Date: September 20th, 2021

DOI: https://doi.org/10.21203/rs.3.rs-491688/v2

License: (c) (i) This work is licensed under a Creative Commons Attribution 4.0 International License. Read Full License 


\section{Abstract}

Background: Multiple reports have described neonatal SARS-CoV-2 infection, including likely in utero transmission and early postnatal infection. Most neonatal infections reported to date have been asymptomatic or mild disease; however, severe cases, including respiratory failure requiring intensive care unit admission, have been described.

Objectives: To describe maternal, pregnancy and infant characteristics among neonates born to women with SARS-CoV-2 infection during pregnancy by neonatal SARS-CoV-2 testing results.

Methods: Using aggregated data from the Surveillance for Emerging Threats to Mothers and Babies Network (SET-NET) from March 29, 2020-August 6, 2021, we identified neonates who were: 1) born to women who were SARS-CoV-2 positive by RT-PCR at any time during their pregnancy, and 2) tested for SARS-CoV-2 by RT-PCR during the birth hospitalization.

Results: Among 25,896 neonates of mothers with SARS-CoV-2-infection, 3,381 (13\%) underwent PCR testing. One hundred thirty-six neonates (4\%) were PCR-positive. Neonates testing positive were born to both symptomatic and asymptomatic women, and $95 \%$ were born to women with infection identified $\leq$ with 14 days of delivery.

Conclusions: While perinatal SARS-CoV-2 infection was uncommon among neonates born to women with SARS-CoV-2 infection during pregnancy, nearly all cases of neonatal infection occurred in pregnant women infected around the time of delivery. These findings underline the need for infection prevention and control measures in delivery and outpatient pediatric settings, as well as counselling for persons who acquire COVID-19 during pregnancy about potential risk to their neonates. Moreover, pregnant people and those wanting to become pregnant should be vaccinated against COVID-19 in order to protect themselves and their infants.

\section{Synopsis}

\section{Study question}

We sought to describe maternal, pregnancy and infant characteristics among neonates born to women with SARS-CoV-2 infection during pregnancy by infant SARS-CoV-2 testing results at birth.

\section{What's already known?}

Multiple reports have described perinatal SARS-CoV-2 infection, including likely in utero transmission and early postnatal infection. Most neonatal infections reported to date have been asymptomatic or mild disease; however, severe cases, including respiratory failure requiring intensive care unit admission, have been described.

\section{What this study adds?}


Among 25,896 neonates of mothers with SARS-CoV-2 infection, 3,381 (13\%) underwent PCR testing with reported results. One hundred thirty-six neonates (4\%) were PCR-positive and were born to both symptomatic and asymptomatic women. Nearly all PCR-positive neonates (95\%) were born to women with infection identified $\leq 14$ days of delivery.

\section{Background}

Severe acute respiratory syndrome coronavirus 2 (SARS-CoV-2) infection can lead to serious coronavirus disease 2019 (COVID-19) in pregnant women, is associated with preterm birth, and poses a potential risk of transmission to the neonate. ${ }^{1-3}$ Multiple reports have described perinatal infection, including likely in utero transmission and early postnatal infection. ${ }^{3,4}$ Most neonatal infections reported to date have been asymptomatic or mild disease; however, severe cases, including respiratory failure requiring intensive care unit (ICU) admission, have been described. ${ }^{5}$ The American Academy of Pediatrics (AAP) and the Centers for Disease Control and Prevention (CDC) recommend all neonates born to women with suspected or confirmed COVID-19 be tested for SARS-CoV-2 by real-time reverse transcription polymerase chain reaction (RT-PCR). ${ }^{6,7}$ However, data describing factors associated with infection among exposed neonates are limited. We sought to describe maternal, pregnancy and infant characteristics among neonates born to women with SARS-CoV-2 infection during pregnancy by neonatal SARS-CoV-2 testing results.

\section{Methods}

\section{Cohort Selection}

The Surveillance for Emerging Threats to Mothers and Babies Network (SET-NET) is a collaboration between CDC and state, local, and territorial health departments to conduct linked-longitudinal surveillance of pregnant women and their infants to understand the effects of emerging and reemerging threats, including COVID-19. ${ }^{8}$ Pregnant women with laboratory confirmed SARS-CoV-2 infection from January 20 to December 31,2020, are retrospectively ascertained through reporting of pregnancy in COVID-19 surveillance and cross-matching of COVID-19 surveillance data with local data systems that contained data on births to assess pregnancy status. The data are then enhanced through a variety of supplemental data sources at the jurisdiction level including, but not limited to, vital statistics, prenatal screening data, administrative datasets, electronic laboratory reporting, and maternal and neonatal medical record review. The aggregated SET-NET data contain variables on maternal demographics and prenatal history, maternal SARS-CoV-2 infection (e.g., timing of infection relative to pregnancy, severity), neonatal characteristics (e.g., gestational age, SARS-CoV-2 testing), among others. This activity was reviewed by $C D C$ and conducted consistent with applicable federal law and policy. ${ }^{9}$ 
Using aggregated SET-NET data compiled through August 6, 2021, we identified neonates who were: 1) born to women who were SARS-CoV-2 RT-PCR positive at any time during their pregnancy, and 2) tested for SARS-CoV-2 by RT-PCR during the birth hospitalization. For neonates with extended hospitalization at birth (>14 days) or whose date of discharge was unknown, only SARS-CoV- 2 tests conducted $\leq 14$ days following birth were included in order to focus on infections from perinatal transmission, rather than community transmission.

\section{Statistical analysis}

We describe neonates by SARS-CoV-2 testing. We focused on SARS-CoV-2 positive neonates and described maternal and birth characteristics, including maternal demographics, maternal COVID-19 disease severity, ${ }^{10}$ timing of maternal infection relative to delivery (determined by date of first positive SARS-CoV-2 or symptom onset, if testing date was missing) and pregnancy complications (e.g., preeclampsia). We assessed neonatal characteristics including gestational age and birthweight. We also described these characteristics among neonates who were tested by SARS-CoV-2 RT-PCR following birth but who tested negative. Because negative results are not reported consistently to all jurisdictions, the group of test-negative neonates are not representative of all neonates testing negative. Therefore, we did not perform statistical comparisons between neonates testing positive and negative for SARS-CoV-2. Nevertheless, the SET-NET data offer a glimpse of the current state of perinatal SARS-CoV-2 RT-PCR testing of neonates, and neonates with negative results are included here for context.

\section{Results}

During March 29, 2020-August 6, 2021, 20 jurisdictions with neonatal testing data available reported on 25,896 liveborn neonates born to 25,487 pregnant women with SARS-CoV-2 infection (Figure). Of liveborn neonates, 22,515 (86.9\%) had no SARS-CoV-2 testing data available, $19(0.1 \%)$ were tested but had indeterminate or unknown results reported, and 3,362 (13.0\%) had positive or negative SARS-CoV-2 RTPCR results reported. The proportion of neonates known to be tested for SARS-CoV-2 increased with maternal trimester of infection, with testing being performed for $1.3 \%(43 / 3424), 2.6 \%(207 / 7,817)$, and $22.4 \%(3,112 / 13,918)$ of neonates born to women with first, second and third trimester infection, respectively. The median time from maternal SARS-CoV-2 infection to delivery was 75 days (IQR:20-143) among neonates not known to be tested and one day (IQR: 0-9) among those tested.

Of the 3,362 neonates with test results, 3,226 had only negative RT-PCR results reported, and 136 (4.0\%) had at least one positive RT-PCR result, representing $0.5 \%[136 / 25,896]$ of livebirths reported. Of these 136 neonates, $90(66.2 \%)$ had only positive RT-PCR results and $46(33.8 \%)$ had both positive and negative results. Of the latter group, 20 neonates tested negative one day before testing positive and nine neonates 
tested positive one day before testing negative. Four neonates had a positive and a negative PCR on the same day.

Maternal demographics and underlying conditions are summarized in the Table. The most frequent maternal race/ethnicity was Hispanic or Latina. Of SARS-CoV-2 positive neonates with date of maternal infection reported $(n=133), 95.5 \%$ were born to women with either initial positive testing or symptom onset occurring $\leq 14$ days before delivery. Six infected neonates were born to women with initial positive SARS-CoV-2 testing $>14$ days before delivery. Three of these women remained SARS-CoV- 2 positive within two days prior to delivery. The percent positivity among neonates tested for SARS-CoV-2 was $0.9 \%$ (6/644), 3.2\% (10/317), 4.6\% (19/409), and 5.0\% (98/1967) for those born to women with infection diagnosed $>14$ days, 7-14 days, 3-6 days and 0-2 days before delivery. Of neonates testing positive whose maternal disease severity was known $(n=85), 42$ (49.4\%) were born to mothers reported to be asymptomatic at time of COVID-19 diagnosis.

Among the 136 neonates testing positive for SARS-CoV-2-positive, $54.1 \%$ were female, $6.9 \%$ were small for gestational age $\left(<10^{\text {th }}\right.$ percentile for sex and gestational age using the INTERGROWTH-21st), and 33 $(24.3 \%)$ were born preterm ( $<37$ weeks gestation), including $8.1 \%(11 / 136)$ born moderate to extremely preterm ( $<34$ weeks). Among neonates born at $\geq 37$ weeks, percent SARS-CoV-2 positivity was $3.8 \%$ $(103 / 2,698)$, compared to $6.0 \%(33 / 546)$ positivity among neonates born preterm. Timing of RT-PCR testing was available for 135 of the neonates with SARS-CoV-2 infection: eight (5.9\%) neonates were first tested on the day of birth, $103(76.3 \%)$ on the second day of life (DOL), and $20(14.8 \%)$ on the third DOL. The timing of RT-PCR testing was similar for neonates testing negative.

\section{Comment}

\section{Principal findings}

SARS-CoV-2 infection among neonates born to women with COVID-19 during pregnancy was uncommon, occurring in $4.0 \%$ of neonates known to be tested for the virus. However, this is likely an overestimate because negative test results are less frequently reported to health departments; the true percentage lies between $4.0 \%$ and $0.5 \%$ (the total neonates testing PCR-positive among all livebirths). While uncommon, neonatal infection was more frequent among neonates born to mothers diagnosed with COVID-19 close to delivery ( $<7$ days) and among neonates born preterm ( $<37$ weeks). Neonatal positivity occurred regardless of maternal symptom status. 
SET-NET is one of the largest cohorts of pregnant women with laboratory confirmed SARS-CoV-2 infections in the U.S. The mother baby linked longitudinal surveillance allows for case identification of infection during pregnancy and follow-up to the birth hospitalization using existing data sources including laboratory reporting.

\section{Limitations of the data}

Limitations of our analysis include the inability to assess route of SARS-CoV-2 transmission (e.g., in utero, peripartum, postnatal), given lack of immunoglobulin-M serology and RT-PCR testing data on sterile specimens (e.g., blood). ${ }^{11}$ We were also not able to assess infection prevention and control (IPC) measures implemented during delivery hospitalization, which may vary. Lastly, testing practices of both pregnant women and their neonates likely varied throughout the course of the pandemic, by facility, and by maternal or neonatal characteristics (e.g., symptom status, ICU admission). ${ }^{12}$

\section{Interpretation}

Previous studies of pregnant women with SARS-CoV-2-infection have suggested higher prevalence of preterm birth, and potentially other adverse outcomes, compared to pregnant women without infection or national baseline estimates. ${ }^{2,13}$ However, much remains unknown about the frequency and risk factors of transmission of SARS-CoV-2 from a pregnant person to their fetus or neonate. Our findings that neonates with positive SARS-CoV-2 testing were born to both symptomatic and asymptomatic women underline the need for IPC measures in delivery and outpatient pediatric settings, as well as counselling for persons who acquire COVID-19 during pregnancy about potential risk to their neonates.

Identification of neonates with SARS-CoV-2 infection will be influenced by testing practices. Although these data are not representative of all perinatal testing practices, they indicate that RT-PCR SARS-CoV-2 testing of neonates born to women with SARS-CoV-2 infection during pregnancy primarily occurred for women with third trimester infection, especially for women with infection identified within 14 days of delivery. These testing patterns are consistent with the idea that transmission from mother to neonate through respiratory droplets is most likely to occur during the mother's infectious period. ${ }^{14}$ Nearly all neonates born to women with infection occurring more than 14 days prior to delivery tested negative; one possible additional explanation is protection against SARS-CoV-2 infection by transplacental transfer of maternal antibodies, ${ }^{15}$ but further studies are needed.

\section{Conclusion}


Previous studies have demonstrated that risk of postnatal transmission from a mother infected with SARS-CoV-2- to neonate is low when appropriate IPC is followed. ${ }^{3}$ The AAP and CDC recommend that mothers with COVID-19 utilize appropriate IPC measures (e.g., masks, hand hygiene) when rooming in with their infants, ${ }^{6,7}$ even if the mother is asymptomatic. Neonates born to women with COVID19 should be tested for SARS-CoV-2, ${ }^{6,7}$ particularly those born to women with infection identified close to delivery or who are born preterm. Future studies that compare infected with SARS-CoV-2 infection to a representative sample of neonates without infection are needed to identify risk factors for neonatal SARS-CoV-2 infection and other neonatal adverse outcomes. Additionally pregnant people and those wanting to become pregnant should be vaccinated against COVID-19 in order to protect themselves and their infants. ${ }^{16}$

\section{Declarations}

\section{Acknowledgements}

We thank the following persons for their contributions to this project: XXX. Finally, we thank the COVID19 Pregnancy and Infant Linked Outcomes Team, the Epidemiology and Surveillance Task Force.

Financial Disclosure: This study was performed as regular work of the Centers for Disease Control and Prevention. This work is supported by the Epidemiology and Laboratory Capacity for Prevention and Control of Emerging Infectious Diseases Cooperative Agreement (CK19-1904) and contractual mechanisms.

Conflict of Interest Disclosures: All authors have no conflicts of interest to disclose.

Disclaimer. The findings and conclusions in this report are those of the authors and do not necessarily represent the views of the Centers for Disease Control and Prevention.

\section{References}

1. Zambrano LD, Ellington S, Strid P, et al. Update: Characteristics of Symptomatic Women of Reproductive Age with Laboratory-Confirmed SARS-CoV-2 Infection by Pregnancy Status - United States, January 22-October 3, 2020. MMWR Morb Mortal Wkly Rep. Published online 2020.

doi:10.15585/mmwr.mm6944e3 
2. Woodworth KR, Olsen EO, Neelam V, et al. Birth and Infant Outcomes Following Laboratory-Confirmed SARS-CoV-2 Infection in Pregnancy - SET-NET, 16 Jurisdictions, March 29-October 14, 2020. MMWR Morb Mortal Wkly Rep. Published online 2020. doi:10.15585/mmwr.mm6944e2

3. Walker KF, O'Donoghue K, Grace N, et al. Maternal transmission of SARS-COV-2 to the neonate, and possible routes for such transmission: A systematic review and critical analysis. BJOG An Int J Obstet Gynaecol. 2020;127(11). doi:10.1111/1471-0528.16362

4. Fenizia $\mathrm{C}$, Biasin $\mathrm{M}$, Cetin I, et al. Analysis of SARS-CoV-2 vertical transmission during pregnancy. Nat Commun. 2020;11(1):5128. doi:10.1038/s41467-020-18933-4

5. Raschetti R, Vivanti AJ, Vauloup-Fellous C, Loi B, Benachi A, De Luca D. Synthesis and systematic review of reported neonatal SARS-CoV-2 infections. Nat Commun. 2020;11(1). doi:10.1038/s41467-02018982-9

6. American Academy of Pediatrics. FAQs: Management of Infants Born to Mothers with Suspected or Confirmed COVID-19. Published September 2020. Accessed August 26, 2021.

https://services.aap.org/en/pages/2019-novel-coronavirus-covid-19-infections/clinical-guidance/faqsmanagement-of-infants-born-to-covid-19-mothers/

7. Centers for Disease Control and Prevention. Evaluation and Management Considerations for Neonates At Risk for COVID-19. Published December 2020. Accessed August 26, 2021. https://www.cdc.gov/coronavirus/2019-ncov/hcp/caring-for-newborns.html

8. Woodworth KR, Reynolds MR, Burkel V, et al. A Preparedness Model for Mother-Baby Linked Longitudinal Surveillance for Emerging Threats. Matern Child Health J. 2021;25(2):198-206. doi:10.1007/s10995-020-03106-y

9. 45 C.F.R. part 46.102(I)(2), 21 C.F.R. part 56; 42 U.S.C. Sect. 241 (d); 5 U.S.C. Sect. 552a; 44 U.S.C. Sect. 3501 et seq. 
10. Galang RR, Newton SM, Woodworth KR, et al. Risk factors for illness severity among pregnant women with confirmed severe acute respiratory syndrome coronavirus 2 infection - Surveillance for Emerging Threats to Mothers and Babies Network, 29 state, local, and territorial health departments, March 29, 2020 -March 5, 2021. Clin Infect Dis. 2021 Jul 15;73(Suppl 1):S17-S23. doi: $10.1093 / \mathrm{cid} / \mathrm{ciab} 432$.

11. World Health Organization. Definition and Categorization of the Timing of Mother-to-Child Transmission of SARS-CoV-2.; 2012. Accessed August 26, 2021. https://www.who.int/publications/i/item/WHO-2019-nCoV-mother-to-child-transmission-2021.1

12. Palmsten $\mathrm{K}$, Vazquez-Benitez G, Kharbanda EO. Point: Uncertainty about estimating the risks of COVID-19 during pregnancy. Paediatr Perinat Epidemiol. 2021:00:1-3. https://doi.org/10.1111/ppe.12773

13. Allotey J, Stallings E, Bonet M, et al. Clinical manifestations, risk factors, and maternal and perinatal outcomes of coronavirus disease 2019 in pregnancy: living systematic review and meta-analysis. BMJ. 2020;370:m3320. Published 2020 Sep 1. doi:10.1136/bmj.m3320

14. Quilty BK, Clifford S, Hellewell J, Russell TW, Kucharski AJ, Flasche S Edmunds WJ. Quarantine and testing strategies in contact tracing for SARS-CoV-2: a modelling study. Lancet Public Health $2021 ; 6(3)$ : e175-e183. https://doi.org/10.1016/ S2468-2667(20)30308-X

15. Flannery DD, Gouma S, Dhudasia MB, et al. Assessment of Maternal and Neonatal Cord Blood SARS-CoV-2 Antibodies and Placental Transfer Ratios. JAMA Pediatr. 2021;175(6):594-600. doi:10.1001/jamapediatrics.2021.0038 
16. Centers for Disease Control and Prevention. COVID-19 Vaccines While Pregnant or Breastfeeding. Updated August 11, 2021. Accessed August 27, 2021. https://www.cdc.gov/coronavirus/2019ncov/vaccines/recommendations/pregnancy.html

\section{Table}


Table. Maternal demographics, maternal disease characteristics, and neonate characteristics by neonate SARS-CoV-2 real-time polymerase chain reaction (RT-PCR) test results - SET-NET, 20 jurisdictions ${ }^{\mathrm{a}}$, March 29, 2020 - August 6, 2021

\begin{tabular}{|c|c|c|}
\hline & \multicolumn{2}{|c|}{$\begin{array}{l}\text { Neonate RT-PCR test results } \\
(\mathrm{N}=3,362)\end{array}$} \\
\hline & Positive & Negative \\
\hline$n(\%)$ & $136(4 \%)$ & $3,226(96 \%)$ \\
\hline \multicolumn{3}{|l|}{ Maternal characteristics } \\
\hline Age in years & $\mathrm{N}=120$ & $\mathrm{~N}=2,966$ \\
\hline Median (IQR) & $\begin{array}{l}29.1(24.4- \\
34.3)\end{array}$ & $\begin{array}{l}29.0(24.7- \\
33.8)\end{array}$ \\
\hline$<25$ & $35(29.2 \%)$ & $785(26.5 \%)$ \\
\hline $25-34$ & $57(47.5 \%)$ & $1,606(54.1 \%)$ \\
\hline$\geq 35$ & $28(23.3 \%)$ & $575(19.4 \%)$ \\
\hline Not reported, $n(\%)$ & $16(11.8 \%)$ & $260(8.1 \%)$ \\
\hline Race/ethnicity & $\mathrm{N}=120$ & $N=3080$ \\
\hline Hispanic or Latina & $65(54.2 \%)$ & $1464(47.5 \%)$ \\
\hline Black, non-Hispanic & $17(14.2 \%)$ & $517(16.8 \%)$ \\
\hline White, non-Hispanic & $32(26.7 \%)$ & $847(27.5 \%)$ \\
\hline Multiple or other race, non-Hispanic & $6(5 \%)$ & $252(8.2 \%)$ \\
\hline Not reported, $n(\%)$ & $16(11.8 \%)$ & $146(4.5 \%)$ \\
\hline \multicolumn{3}{|l|}{ Underlying conditions } \\
\hline & $\mathrm{N}=121$ & $\mathrm{~N}=2895$ \\
\hline \multirow[t]{2}{*}{ Any underlying condition ${ }^{\mathrm{b}}$} & $58(47.9 \%)$ & $1343(46.4 \%)$ \\
\hline & $\mathrm{N}=113$ & $\mathrm{~N}=2476$ \\
\hline Hypertensive disorders of pregnancy ${ }^{\mathrm{C}}$ & $17(15.0 \%)$ & $326(13.2 \%)$ \\
\hline Trimester of maternal infection & $\mathrm{N}=135$ & $\mathrm{~N}=3208$ \\
\hline First & $0(0 \%)$ & $43(1.3 \%)$ \\
\hline Second & $1(0.7 \%)$ & $203(6.3 \%)$ \\
\hline Third & $134(99.3 \%)$ & $2962(92.3 \%)$ \\
\hline Not reported, $n(\%)$ & $1(0.7 \%)$ & $18(0.6 \%)$ \\
\hline
\end{tabular}




\begin{tabular}{|c|c|c|}
\hline $\begin{array}{l}\text { Timing of mother's first positive RT-PCR test (days prior to } \\
\text { delivery) }\end{array}$ & $\mathrm{N}=133$ & $N=3204$ \\
\hline Median (IQR) & $1(0-3)$ & $1(0-9)$ \\
\hline$>14$ & $6(4.5 \%)$ & $638(19.9 \%)$ \\
\hline $7-14$ & $10(7.5 \%)$ & $307(9.6 \%)$ \\
\hline $3-6$ & $19(14.3 \%)$ & $390(12.2 \%)$ \\
\hline $0-2$ & $98(73.7 \%)$ & $1869(58.3 \%)$ \\
\hline Not reported, $n(\%)$ & $4(2.9 \%)$ & $22(0.7 \%)$ \\
\hline Maternal disease severity & $\mathrm{N}=85$ & $\mathrm{~N}=1753$ \\
\hline Asymptomatic & $42(49.4 \%)$ & $732(41.8 \%)$ \\
\hline Mild & $28(32.9 \%)$ & $674(38.4 \%)$ \\
\hline Moderate/severe & $9(10.6 \%)$ & $252(14.4 \%)$ \\
\hline Critical & $6(7.1 \%)$ & $95(5.4 \%)$ \\
\hline Not enough information, $n$ (\%) & $51(37.5 \%)$ & $1473(45.7 \%)$ \\
\hline Delivery type & $\mathrm{N}=132$ & $N=2958$ \\
\hline Vaginal & $72(54.5 \%)$ & $1863(63 \%)$ \\
\hline Cesarean & $60(45.5 \%)$ & $1095(37 \%)$ \\
\hline Not reported, n (\%) & $4(2.9 \%)$ & $268(8.3 \%)$ \\
\hline \multicolumn{3}{|l|}{ Infant characteristics } \\
\hline Gestational age at birth & $\mathrm{N}=136$ & $N=3108$ \\
\hline Median weeks (IQR) & $\begin{array}{l}38.7(37.1- \\
39.6)\end{array}$ & $\begin{array}{l}39.0(37.6- \\
40.0)\end{array}$ \\
\hline Term ( $\geq 37$ wks) & $103(75.7 \%)$ & $2595(83.5 \%)$ \\
\hline Preterm (<37 wks) & $33(24.3 \%)$ & $513(16.5 \%)$ \\
\hline Late preterm (34-36 wks) & $22(16.2 \%)$ & $347(11.2 \%)$ \\
\hline Moderate to extremely preterm ( $<34$ wks) & $11(7.4 \%)$ & $166(4.6 \%)$ \\
\hline Not reported, $n(\%)$ & $0(0 \%)$ & $111(3.4 \%)$ \\
\hline Neonate Sex & $\mathrm{N}=135$ & $N=3224$ \\
\hline Female & $73(54.1 \%)$ & $1579(49 \%)$ \\
\hline Male & $62(45.9 \%)$ & $1645(51 \%)$ \\
\hline
\end{tabular}




\begin{tabular}{|lll|}
\hline Not reported, $n(\%)$ & $1(0.7 \%)$ & $2(0.1 \%)$ \\
\hline Birthweight & $\mathbf{N}=\mathbf{1 3 1}$ & $\mathbf{N}=\mathbf{2 7 6 2}$ \\
\hline Low birthweight $(<2500 \mathrm{~g})$ & $18(13.7 \%)$ & $319(11.5 \%)$ \\
\hline Small for gestational age & $\mathbf{N}=\mathbf{1 3 0}$ & $\mathbf{N}=\mathbf{2 5 0 2}$ \\
\hline Not reported, $n$ (\%) & $9(6.9 \%)$ & $192(7.7 \%)$ \\
\hline Neonatal intensive care unit admission & $6(4.4 \%)$ & $724(22.4 \%)$ \\
\hline Admitted during birth hospitalization & $\mathbf{N}=\mathbf{1 2 2}$ & $\mathbf{N}=\mathbf{2 4 9 5}$ \\
\hline Not reported, $n$ (\%) & $42(34.4 \%)$ & $577(23.1 \%)$ \\
\hline Day of first RT-PCR test following birth, in days of life & $8(6.2 \%)$ & $628(20.1 \%)$ \\
\hline 1 & $\mathbf{N}=135$ & $\mathbf{N}=\mathbf{3 1 9 2}$ \\
\hline 2 & $8(5.9 \%)$ & $189(5.9 \%)$ \\
\hline 3 & $103(76.3 \%)$ & $2357(73.8 \%)$ \\
\hline$\geq 4$ & $20(14.8 \%)$ & $490(15.4 \%)$ \\
\hline & $4(3 \%)$ & $156(4.9 \%)$ \\
\hline
\end{tabular}

a Including California [excluding Los Angeles County], Georgia, Houston, Kansas, Los Angeles County, Massachusetts, Maryland, Michigan, Minnesota, Missouri, Nebraska, Nevada, New Jersey, New York [excluding New York City], North Dakota, Pennsylvania [excluding Philadelphia], Puerto Rico, Tennessee, Vermont, and Washington

bIncludes cardiovascular disease, chronic hypertension, chronic lung disease, diabetes mellitus (type 1 or type 2), immunosuppression, obesity (body mass index $\geq 30 \mathrm{~kg} / \mathrm{m}^{2}$ ).

'Inclusive of preeclampsia, eclampsia, or HELLP (hemolysis, elevated liver enzymes and low platelet) syndrome. Does not include chronic hypertension. Hypertensive disorders of pregnancy were only considered underlying conditions for women with third trimester infection.

${ }^{d}$ Categories of disease severity were based on modified National Institute of Health and World Health Organization criteria as described in Galang et al ${ }^{9}$. Women were considered asymptomatic if reported as having an absence of symptoms using a symptom status variable rather than solely absence of individual symptoms reported. Criteria were applied to classify severity using submitted data including symptoms, intensive care unit admission, invasive ventilation, use of COVID-19 therapies, complications associated with COVID-19, and death. 
${ }^{e}$ Defined as weight $<10$ th percentile for sex (presumed female if missing) and gestational age using the INTERGROWTH-21st online percentile calculator http://intergrowth21.ndog.ox.ac.uk. Some gestational ages were reported in completed weeks only (rather than in weeks and days).

\section{Figures}

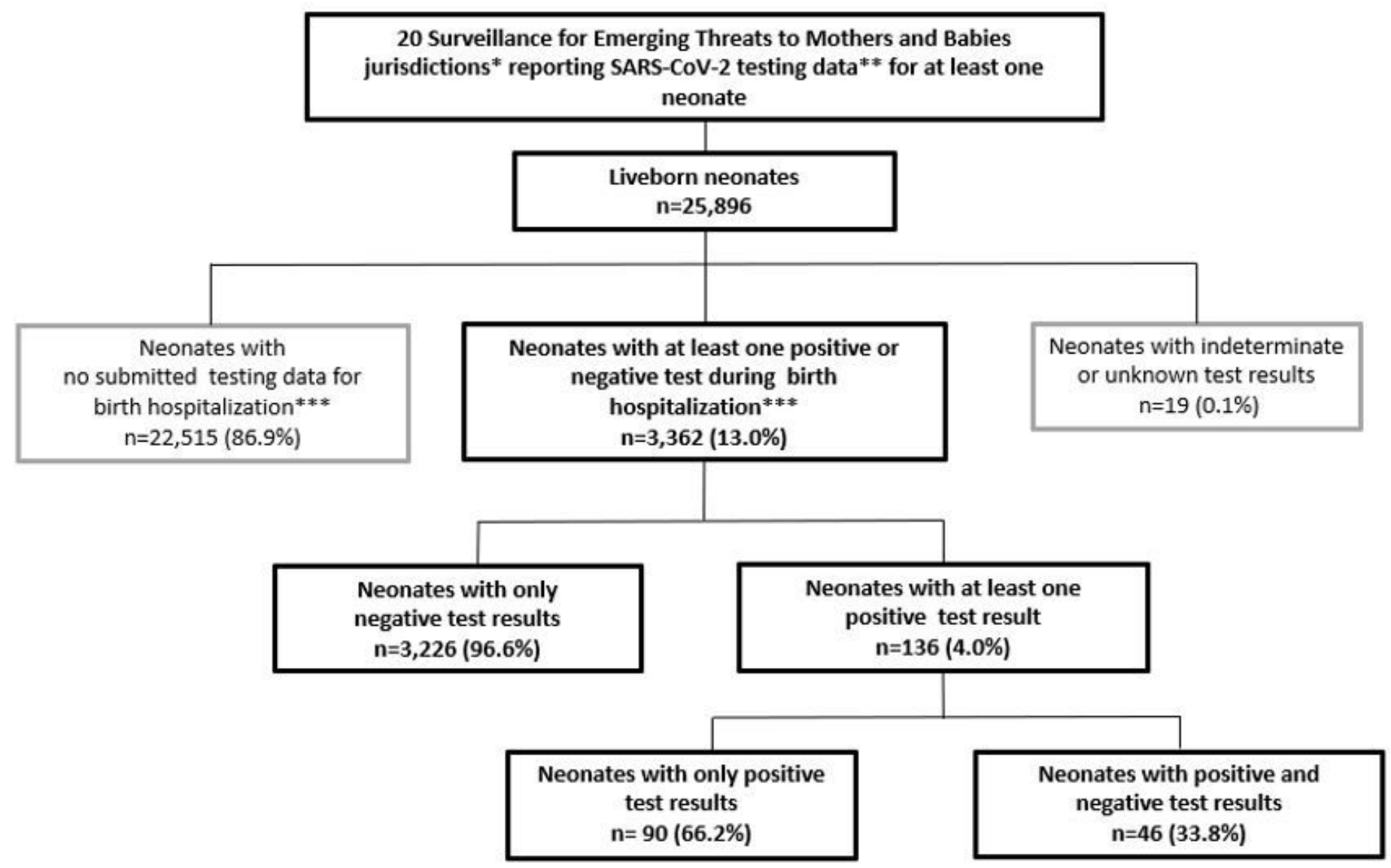

Figure 1

* 20 jurisdictions reporting SARS-CoV-2 lab data for at least one neonate: California [excluding Los Angeles County], Georgia, Houston, Kansas, Los Angeles County, Massachusetts, Maryland, Michigan, Minnesota, Missouri, Nebraska, Nevada, New Jersey, New York [excluding New York City], North Dakota, Pennsylvania [excluding Philadelphia], Puerto Rico, Tennessee, Vermont, Washington** All testing data herein was SARS-CoV-2 by real-time reverse transcription polymerase chain reaction (RT-PCR) *** The birth hospitalization period included only SARS-CoV- 2 tests conducted $\leq 14$ days following birth. 